for

\title{
The Effect of lodine on the Peroxidation of Carbonyl Compounds
}

\author{
Katja Žmitek, ${ }^{a}$ Marko Zupan, ${ }^{b}$ Stojan Stavber, ${ }^{a}$ Jernej Iskra ${ }^{\star a}$ \\ ${ }^{a}$ Laboratory of Organic and Bioorganic Chemistry, “Jožef Stefan” Institute, Jamova 39, Ljubljana, Slovenia. \\ ${ }^{b}$ Faculty of Chemistry and Chemical Technology, University of Ljubljana, Aškerčeva 5, Ljubljana, Slovenia. \\ E-mail: jernej.iskra@ijs.si
}

\section{Table of contents:}

S2 General procedure for synthesis of DHPs from ketones and aromatic aldehydes

S4 Synthesis of hydroperoxyketals and hydroperoxyacetals

S5 Synthesis of bis-perethers

S5 General procedure for synthesis of hydroperoxyhemiacetals

S6 Interconversions between dihydroperoxide $\mathbf{2}$ and hydroperoxyketal 34

S6 References

S7 $\quad{ }^{1} \mathrm{H}$ NMR spectra of dihydroperoxide 20 (react. mixture)

S8 $\quad{ }^{13} \mathrm{C}$ NMR spectra of dihydroperoxide 20 (react. mixture)

S9 $\quad{ }^{1} \mathrm{H}$ NMR spectra of peroxyketals $\mathbf{3 6}$

S10 ${ }^{13} \mathrm{C}$ NMR spectra of peroxyketals $\mathbf{3 6}$

S11 ${ }^{1} \mathrm{H}$ NMR spectra of hydroperoxyketal $\mathbf{3 7}$ (react. mixture)

S12 ${ }^{1} \mathrm{H}$ NMR spectra of bis-perether $\mathbf{3 8}$

S13 ${ }^{13}$ C NMR spectra of bis-perether 38

S14 ${ }^{1}$ H NMR spectra of dihydroperoxide 40b

S15 ${ }^{13}$ C NMR spectra of dihydroperoxide 40b

S16 ${ }^{1}$ H NMR spectra of hydroperoxyhemiacetal $\mathbf{4 5 a}$

S17 ${ }^{13}$ C NMR spectra of hydroperoxyhemiacetal 45a

S18 ${ }^{1} \mathrm{H}$ NMR spectra of peroxyhemiacetal 47a

S19 ${ }^{13}$ C NMR spectra of peroxyhemiacetal 47a 


\section{General remarks:}

Ketones, $\mathrm{I}_{2}$, solvents and ${ }^{t} \mathrm{BuOOH}$ and $30 \% \mathrm{H}_{2} \mathrm{O}_{2}$ were obtained from commercial sources and were used as received. Silica gel 60 (70-230 mesh ASTM) was used for column chromatography.

${ }^{1} \mathrm{H}$ and ${ }^{13} \mathrm{C}$ spectra were obtained on a $300 \mathrm{MHz}$ spectrometer with TMS and $\mathrm{CDCl}_{3}$ as internal standards. Standard $\mathrm{KBr}$ pellet procedures were used to obtain IR spectra of solids, while a film of neat material was used for liquid products. Absorptions are reported in $\mathrm{cm}^{-1}$ $\left(v_{\max }\right)$. Mass spectra was acquired with electron-spray ionization $(8 \mathrm{kV}$ spray needles, $\mathrm{NH}_{4} \mathrm{OAc}$ ). Active oxygen content was determined by iodometric titration. ${ }^{1}$

Caution. Although we have encountered no difficulties in working with these peroxides, routine precautions (shields, fume hoods, avoidance of transition metal salts) should be observed whenever possible, as organic peroxides are potentially hazardous compounds.

\section{Synthesis of gem-dihydroperoxides.}

General procedure for synthesis of DHPs from ketones and aromatic aldehydes. Preparation of 2 is typical. To a solution of $0.1 \mathrm{mmol}$ of $\mathrm{I}_{2}(25.4 \mathrm{mg})$ and $4 \mathrm{mmol}$ of $30 \%$ $\mathrm{H}_{2} \mathrm{O}_{2}(0.45 \mathrm{~mL})$ in $10 \mathrm{~mL}$ of acetonitrile, $1 \mathrm{mmol}(154 \mathrm{mg})$ of $4-t$-Bu-cyclohexanone 1 was added and the solution stirred at $22^{\circ} \mathrm{C}$ for 5 hours. The reaction mixture was concentrated under reduced pressure (cca. $20 \mathrm{mmHg}$ ), dichloromethane $(10 \mathrm{~mL})$ added and the solution dried over $\mathrm{Na}_{2} \mathrm{SO}_{4}$. The solvent was evaporated and product 2 isolated by column chromatography $\left(\mathrm{SiO}_{2}, \mathrm{CH}_{2} \mathrm{Cl}_{2}: \mathrm{EtOAc}=8: 2\right)$.

4-tert-butylcyclohexane-1,1-diyl dihydroperoxide $2:^{2}$ 185mg (91\%), white solid, mp 79$81^{\circ} \mathrm{C},{ }^{1} \mathrm{H}$ NMR $\left(\mathrm{CDCl}_{3}\right): 0.87(\mathrm{~s}, 9 \mathrm{H}), 1.06(\mathrm{~m}, 1 \mathrm{H}), 1.25(\mathrm{~m}, 2 \mathrm{H}), 1.46(\mathrm{~m}, 2 \mathrm{H}), 1.72(\mathrm{bd}, 2 \mathrm{H}$, $J=12 \mathrm{~Hz}), 2.30(\mathrm{bd}, 2 \mathrm{H}, J=12 \mathrm{~Hz}), 9.38(\mathrm{bs}, 2 \mathrm{H}) ;{ }^{13} \mathrm{C} \mathrm{NMR}\left(\mathrm{CDCl}_{3}\right): 23.2,27.6,29.7,32.3$, $47.4,111.0$.

4-Methylcyclohexane-1,1-diyl dihydroperoxide $6(98 \%):^{3}$

white solid, $\mathrm{mp} 135^{\circ} \mathrm{C}$ with decomposition,

${ }^{1} \mathrm{H} \mathrm{NMR}\left(\mathrm{CDCl}_{3}, \mathrm{ppm}\right): 0.95(\mathrm{~d}, 6.4 \mathrm{~Hz}, 3 \mathrm{H}), 1.1-1.80(\mathrm{~m}, 7 \mathrm{H}), 2.2(\mathrm{~m}, 2 \mathrm{H}), 9.25$ (bs, 2H);

${ }^{13} \mathrm{C} \mathrm{NMR}\left(\mathrm{CDCl}_{3}, \mathrm{ppm}\right): 21.4,29.0,30.6,31.6,110.9$

MS-ESI $\left(\mathrm{NH}_{4}^{+}\right): 180\left(\mathrm{MNH}_{4}{ }^{+}\right)$

\section{3-Methylcyclohexane-1,1-diyl dihydroperoxide $8(93 \%):{ }^{4}$}

colorless oil,

${ }^{1} \mathrm{H}$ NMR $\left(\mathrm{CDCl}_{3}, \mathrm{ppm}\right): 0.81-1.01(\mathrm{~m}, 4 \mathrm{H}), 1.12(\mathrm{t}, 1 \mathrm{H}, J=13 \mathrm{~Hz}), 1.26-1.79(\mathrm{~m}, 5 \mathrm{H}), 2.23$

(bd, 2H, J=13 Hz), 9.28 (bs, 2H); ${ }^{13} \mathrm{C} \mathrm{NMR}\left(\mathrm{CDCl}_{3}, \mathrm{ppm}\right): 21.9,22.0,29.0,29.0,34.0,37.4$, 111.4; IR: 3440, 11551005.

MS-ESI $\left(\mathrm{NH}_{4}^{+}\right): 185\left(\mathrm{MNa}^{+}\right)$

2-Methylcyclohexane-1,1-diyl dihydroperoxide $10(80 \%):^{5}$

colorless oil,

${ }^{1} \mathrm{H} \mathrm{NMR}\left(\mathrm{CDCl}_{3}, \mathrm{ppm}\right): 1.07$ (d, 3H, $\left.J=7 \mathrm{~Hz}\right), 1.37-1.80(\mathrm{~m}, 7 \mathrm{H}), 1.89-2.01(\mathrm{~m}, 1 \mathrm{H}), 2.20-$

$2.34(\mathrm{~m}, 1 \mathrm{H}), 8.83(\mathrm{~s}, 1 \mathrm{H}), 8.89(\mathrm{~s}, 1 \mathrm{H}) ;{ }^{13} \mathrm{C} \mathrm{NMR}\left(\mathrm{CDCl}_{3}\right): 14.4,20.2,22.4,24.8,29.5,32.0$, 113.2 . 
Cyclopentane-1,1-diyl dihydroperoxide $12(70 \%):^{6}$

colorless oil,

${ }^{1} \mathrm{H}$ NMR $\left(\mathrm{CDCl}_{3}, \mathrm{ppm}\right)$ : 1.63-1.82 (m, 4H), 1.88-2.06 (m, 4H), 9.69 (bs, 2H); ${ }^{13} \mathrm{C} \mathrm{NMR}$

$\left(\mathrm{CDCl}_{3}, \mathrm{ppm}\right): 24.5,33.1,122.2$.

Cycloheptane-1,1-diyl dihydroperoxide $14(90 \%):^{3}$

white solid, $\mathrm{mp} 60-61{ }^{\circ} \mathrm{C}$

${ }^{1} \mathrm{H} \mathrm{NMR}\left(\mathrm{CDCl}_{3}, \mathrm{ppm}\right): 1.50(\mathrm{~s}, 8 \mathrm{H}), 1.87-2.11(\mathrm{~m}, 4 \mathrm{H}), 9.64(\mathrm{~s}, 2 \mathrm{H}) ;{ }^{13} \mathrm{C} \mathrm{NMR}\left(\mathrm{CDCl}_{3}, \mathrm{ppm}\right)$ : 22.6, 22.7, 29.8, 30.0, 32.4, 33.0, 116.2.

Cyclododecane-1,1-diyl dihydroperoxide $16(60 \%):^{3}$

white solid, $\mathrm{mp} 137-138^{\circ} \mathrm{C}$ with decomposition

${ }^{1} \mathrm{H}$ NMR (DMSO-d6, ppm): 1.20-1.60 (m, 22H), 10.81 (s, 2H); ${ }^{13} \mathrm{C}$ NMR (DMSO-d6, ppm): $18.9,21.5,21.8,25.6,25.8,25.9,112.3$.

Adamantane-2,2-diyl dihydroperoxide $18(92 \%):^{3}$

mp $147-148^{\circ} \mathrm{C}$

${ }^{1} \mathrm{H}$ NMR $\left(\mathrm{CDCl}_{3}, \mathrm{ppm}\right): 1.64-2.39(\mathrm{~m}, 14 \mathrm{H}), 8.80(\mathrm{~s}, 2 \mathrm{H}) ;{ }^{13} \mathrm{C} \mathrm{NMR}\left(\mathrm{CDCl}_{3}, \mathrm{ppm}\right): 27.0,31.2$, 33.7, 37.0, 112.7.

3,3-Dihydroperoxyandrostane-17-on $22(77 \%)^{4}$

white solid, $\mathrm{mp} 161-161.5^{\circ} \mathrm{C}$ with decomposition

${ }^{1} \mathrm{H}$ NMR (CDCl $\left.{ }_{3}, \mathrm{ppm}\right):$ 0.76-2.50 (m, 22H), 0.84 (s, 3H), 0.87 (s, 3H), 8.35 (s, 1H), 8.53 (s, $1 \mathrm{H}) ;{ }^{13} \mathrm{C}$ NMR $\left(\mathrm{CD}_{3} \mathrm{CN}, \mathrm{ppm}\right): 11.9,14.2,21.3,22.4,26.5,28.8,31.7,32.6,32.7,35.8,35.9$, 36.3, 36.8, 43.2, 48.5, 52.2, 55.2, 110.4, 221.4; IR: 3400, 3280, 1700, 1060, 1030, 980.

5 $\alpha$-Cholestane-3 $\alpha, 3 \beta$-dihydroperoxide $25:^{7}$

white solid: ${ }^{1} \mathrm{H}$ NMR $\left(\mathrm{CDCl}_{3}, \mathrm{ppm}\right)$ : 0.65 (s, 3H), 0.81 (s, 3H), 0.86 and 0.87 (both d, $J=6$ $\mathrm{Hz}, 3 \mathrm{H}), 0.90$ (d, J= $6 \mathrm{~Hz}, 3 \mathrm{H}), 8.0$ (bs, 1H), 8.08 (bs, 1H).

Decane-3,3-diyl dihydroperoxide $4(91 \%):^{4}$

white solid, mp $30.1-30.5^{\circ} \mathrm{C}$,

$\left.{ }^{1} \mathrm{H} \mathrm{NMR}\left(\mathrm{CDCl}_{3}, \mathrm{ppm}\right): 0.88(\mathrm{t}, 3 \mathrm{H}, J=7 \mathrm{~Hz}), 0.96(\mathrm{t}, 3 \mathrm{H}, J=7 \mathrm{~Hz}),\right), 1.21-1.46(\mathrm{~m}, 10 \mathrm{H})$, 1.60-1.80 (m, 4H), 8.50 (s, 2H) $;{ }^{13} \mathrm{C} \mathrm{NMR}\left(\mathrm{CDCl}_{3}, \mathrm{ppm}\right): 8.0,14.1,22.3,22.6,23.5,28.7$, 29.1, 29.7, 31.7, 114.7; IR: 3440, 1150, 1130.

MS-ESI $\left(\mathrm{NH}_{4}^{+}\right): 224\left(\mathrm{MNH}_{4}^{+}\right)$

Nonane-2,2-diyl dihydroperoxide $27(96 \%):^{4}$

colorless oil,

${ }^{1} \mathrm{H}$ NMR (CDCl 3 , ppm): 0.88 (t, 3H, J=7 Hz), 1.17-1.50 (m, 13H), 1.70-1.80 (m, 2H), 8.97(s, $2 \mathrm{H}) ;{ }^{13} \mathrm{C} \mathrm{NMR}\left(\mathrm{CDCl}_{3}, \mathrm{ppm}\right): 14.1,17.8,22.6,23.9,29.1,29.7,31.7,33.0,112.7$; IR: 3440, 1130, 1095.

MS-ESI $\left(\mathrm{NH}_{4}{ }^{+}\right): 210\left(\mathrm{MNH}_{4}{ }^{+}\right)$

Nonane-5,5-diyl dihydroperoxide $29(78 \%):^{8}$

colorless oil,

${ }^{1} \mathrm{H}$ NMR $\left(\mathrm{CDCl}_{3}, \mathrm{ppm}\right): 0.92$ (t, 6H, $\left.J=7 \mathrm{~Hz}\right), 1.27-1.43$ (m, 8H), 1.61-1.75 (m, 4H), 9.12 (bs, $2 \mathrm{H}) ;{ }^{13} \mathrm{C} \mathrm{NMR}\left(\mathrm{CDCl}_{3}, \mathrm{ppm}\right): 13.9,22.8,25.7,28.9,114.3$ 
5-Methylheptane-3,3-diyl dihydroperoxide $31(50 \%):^{4}$

colorless oil,

${ }^{1} \mathrm{H}$ NMR $\left(\mathrm{CDCl}_{3}, \mathrm{ppm}\right): 0.89$ (t, 3H, $\left.J=7 \mathrm{~Hz}\right), 0.97$ (t, 6H, $\left.J=7 \mathrm{~Hz}\right), 1.14-1.32(\mathrm{~m}, 1 \mathrm{H}), 1.32-$

$1.92(\mathrm{~m}, 6 \mathrm{H}), 8.82(\mathrm{~s}, 2 \mathrm{H}) ;{ }^{13} \mathrm{C} \mathrm{NMR}\left(\mathrm{CDCl}_{3}, \mathrm{ppm}\right): 8.1,11.3,19.9,22.6,30.2,30.5,34.7$,

115.1; IR: 3330, 1150, 1130.

MS-ESI $\left(\mathrm{NH}_{4}^{+}\right): 201\left(\mathrm{MNa}^{+}\right)$

1-Phenylethane-1,1-diyl dihydroperoxide $33(16 \%):^{4}$

white solid, $\mathrm{mp} 76.8-77.4^{\circ} \mathrm{C}$

${ }^{1} \mathrm{H} \mathrm{NMR}\left(\mathrm{CDCl}_{3}, \mathrm{ppm}\right): 1.70(\mathrm{~s}, 3 \mathrm{H}), 7.31-7.41(\mathrm{~m}, 3 \mathrm{H}), 7.46-7.58(\mathrm{~m}, 2 \mathrm{H}), 9.31(\mathrm{~s}, 2 \mathrm{H}) ;{ }^{13} \mathrm{C}$ NMR $\left(\mathrm{CDCl}_{3}\right.$, ppm): 23.1, 111.4, 125.7, 128.4, 128.6, 138.2; IR: 3348, 3219, 1142, 1123, 855.

Phenylmethylene dihydroperoxide 40a $(55 \%):^{9}$

colorless oil

${ }^{1} \mathrm{H}$ NMR $\left(\mathrm{CDCl}_{3}, \mathrm{ppm}\right): 6.32(\mathrm{~s}, 1 \mathrm{H}), 7.20-7.52(\mathrm{~m}, 5 \mathrm{H}), 9.60(\mathrm{~s}, 2 \mathrm{H}) ;{ }^{13} \mathrm{C} \mathrm{NMR}\left(\mathrm{CDCl}_{3}\right.$, ppm): 109.9, 127.0, 128.4, 129.7, 132.3.

(4-Methoxyphenyl)methylene dihydroperoxide 40c (76\%): ${ }^{4}$

light yellow oil (unstable compound, partial decomposition at $4^{\circ} \mathrm{C}$ after few hours)

${ }^{1} \mathrm{H} \mathrm{NMR}\left(\mathrm{CDCl}_{3}, \mathrm{ppm}\right): 3.80(\mathrm{~s}, 3 \mathrm{H}), 6.27(\mathrm{~s}, 1 \mathrm{H}), 6.88(\mathrm{~d}, 2 \mathrm{H}, J=9 \mathrm{~Hz}), 7.34(\mathrm{~d}, 2 \mathrm{H}, J=9$

$\mathrm{Hz}), 9.41(\mathrm{~s}, 2 \mathrm{H}) ;{ }^{3} \mathrm{C}$ NMR $\left(\mathrm{CDCl}_{3}, \mathrm{ppm}\right): 55.3,110.1,113.9,124.4,128.5,160.5$. IR: 3360 , 1230, 1170, 1040, 830, 730 .

\section{Tetrahydro-2H-pyran-4,4-diyl dihydroperoxide 20 :}

To a solution of $0.1 \mathrm{mmol}$ of $\mathrm{I}_{2}(25.4 \mathrm{mg})$ and $4 \mathrm{mmol}$ of $30 \% \mathrm{H}_{2} \mathrm{O}_{2}(0.45 \mathrm{~mL})$ in $2 \mathrm{~mL}$ of acetonitrile, $1 \mathrm{mmol}(100 \mathrm{mg})$ of tetrahydro- $4 \mathrm{H}$-pyranone 19 was added and the solution stirred at $22^{\circ} \mathrm{C}$ for 4 hours. The reaction mixture was then concentrated under reduced pressure (cca. $20 \mathrm{mmHg}$ ), ethylacetate $(10 \mathrm{~mL}$ ) was added and the solution dried over $\mathrm{Na}_{2} \mathrm{SO}_{4}$. The solvent was evaporated and $140 \mathrm{mg}$ of reaction mixture isolated. Conversion to DHP 20 determined from ${ }^{1} \mathrm{H}$ NMR of the reaction mixture was $89 \%$. DHP 20 was not stable and its structure was deduced by comparison of spectroscopic data with its stable perether 38 . Data for 20: ${ }^{1} \mathrm{H}$ NMR $\left(\mathrm{CDCl}_{3}\right)$ : 1.81-2.11 (m, 4H), 3.70-3.85 (m, 4H), $7.87(\mathrm{bs}, 2 \mathrm{H}) ;{ }^{13} \mathrm{C}$ $\operatorname{NMR}\left(\mathrm{CDCl}_{3}\right): 31.9,64.7,103.1$.

\section{Synthesis of hydroperoxyketals and hydroperoxyacetals. \\ 4-tert-Butyl-1-methoxy-cyclohexyl-hydroperoxide $34:^{10}$}

To a solution of $0.05 \mathrm{mmol}$ of $\mathrm{I}_{2}(12.7 \mathrm{mg})$ and $1 \mathrm{mmol}$ of $30 \% \mathrm{H}_{2} \mathrm{O}_{2}(0.12 \mathrm{~mL})$ in $2 \mathrm{~mL}$ of methanol, $1 \mathrm{mmol}(154 \mathrm{mg})$ of 4-t-Bu-cyclohexanone 1 was added and the solution stirred at $22^{\circ} \mathrm{C}$ for 24 hours. The mixture was then poured into water and theproducts extracted with dichloromethane. The organic phase was dried over $\mathrm{Na}_{2} \mathrm{SO}_{4}$ and the solvent evaporated under reduced pressure (cca. $20 \mathrm{mmHg}$ ). The product was isolated by column chromatography $\left(\mathrm{SiO}_{2}, \mathrm{CH}_{2} \mathrm{Cl}_{2}:\right.$ EtOAc=9:1) resulting in $145 \mathrm{mg}$ (72\%) of white solid, 3: 2 mixture of diastereomers, major diastereomer (from mixture): ${ }^{1} \mathrm{H}$ NMR $\left(\mathrm{CDCl}_{3}, \mathrm{ppm}\right): 0.86(\mathrm{~s}, 9 \mathrm{H})$, 0.92-1.50 (m, 5H), $1.69(\mathrm{~m}, 2 \mathrm{H}), 2.03-2.38(\mathrm{~m}, 2 \mathrm{H}), 3.34(\mathrm{~s}, 3 \mathrm{H}), 7.89(\mathrm{~s}, 1 \mathrm{H}) ;{ }^{13} \mathrm{C} \mathrm{NMR}$ $\left(\mathrm{CDCl}_{3}, \mathrm{ppm}\right): 23.6,27.6,30.9,32.3,47.4,48.5,105.4$.

minor diastereomer (from mixture): ${ }^{1} \mathrm{H}$ NMR $\left(\mathrm{CDCl}_{3}\right): 0.86(\mathrm{~s}, 9 \mathrm{H}), 0.92-1.50(\mathrm{~m}, 5 \mathrm{H}), 1.69$ $(\mathrm{m}, 2 \mathrm{H}), 2.03-2.38(\mathrm{~m}, 2 \mathrm{H}), 3.30(\mathrm{~s}, 3 \mathrm{H}), 7.89(\mathrm{~s}, 1 \mathrm{H}) ;{ }^{13} \mathrm{C} \mathrm{NMR}\left(\mathrm{CDCl}_{3}\right)$ : 23.4, 27.6, 30.9, $31.4,47.5,48.2,105.7$. 


\section{4-tert-Butyl-1-ethoxycyclohexyl hydroperoxide 37}

To a solution of $0.1 \mathrm{mmol}$ of $\mathrm{I}_{2}(25.4 \mathrm{mg})$ and $1.1 \mathrm{mmol}$ of $30 \% \mathrm{H}_{2} \mathrm{O}_{2}(0.13 \mathrm{~mL})$ in $4 \mathrm{~mL}$ of ethanol, $1 \mathrm{mmol}(154 \mathrm{mg})$ of 4-t-Bu-cyclohexanone 1 was added and the solution stirred at $22^{\circ} \mathrm{C}$ for 24 hours. The reaction mixture was concentrated under reduced pressure (cca. 20 $\mathrm{mmHg}$ ), dichloromethane $(10 \mathrm{~mL})$ added and the solution dried over $\mathrm{Na}_{2} \mathrm{SO}_{4}$. The solvent was evaporated and obtained $204 \mathrm{mg}$ of mixture of starting substrate and 37. Identification was made by comparing NMR spectroscopic data of the reaction mixture with analogous product 34, but further attempts for its isolation failed as it decomposed to the starting ketone. ${ }^{1} \mathrm{H} \mathrm{NMR}\left(\mathrm{CDCl}_{3}\right): 0.86(\mathrm{~s}, 9 \mathrm{H}), 0.95-1.56(\mathrm{~m}, 8 \mathrm{H}), 1.58-1.77(\mathrm{~m}, 2 \mathrm{H}), 2.0-2.50(\mathrm{~m}, 2 \mathrm{H}), 3.59$ $(2 \mathrm{q}, J=7 \mathrm{~Hz}, 2 \mathrm{H}), 7.94(\mathrm{bs}, 1 \mathrm{H}) ;{ }^{13} \mathrm{C}$ NMR $\left(\mathrm{CDCl}_{3}\right)$ : characteristic signals 105.7 and 105.4

\section{Synthesis of bis-perethers.}

\section{4,4-Bis(tert-butylperoxy)tetrahydro- $2 H$-pyran 38 :}

To a solution of $0.1 \mathrm{mmol}$ of $\mathrm{I}_{2}(25.4 \mathrm{mg})$ and $2 \mathrm{mmol}$ of $60 \%{ }^{t} \mathrm{BuOOH}$ decane solution $(0.72$ $\mathrm{mL})$ in $2 \mathrm{~mL}$ of acetonitrile, $1 \mathrm{mmol}(100 \mathrm{mg})$ of tetrahydro- $4 H$-pyranone 19 was added and solution stirred at $22^{\circ} \mathrm{C}$ for 4 hours. The reaction mixture was concentrated under reduced pressure (cca. $20 \mathrm{mmHg}$ ) product was isolated by column chromatography $\left(\mathrm{SiO}_{2}\right.$, hexane:EtOAc=95:5); $134 \mathrm{mg}(51 \%)$ of colorless oil. ${ }^{1} \mathrm{H} \mathrm{NMR}\left(\mathrm{CDCl}_{3}\right): 1.26(\mathrm{~s}, 18 \mathrm{H}), 1.95$ $(\mathrm{t}, J=5 \mathrm{~Hz}, 4 \mathrm{H}), 3.71(\mathrm{t}, J=5 \mathrm{~Hz}, 4 \mathrm{H}) ;{ }^{13} \mathrm{C} \mathrm{NMR}\left(\mathrm{CDCl}_{3}\right): 26.7,31.6,64.8,79.4,104.3$; IR:

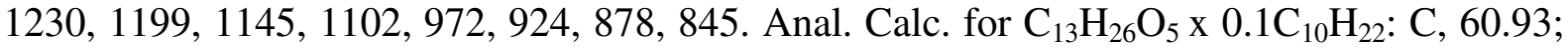
$\mathrm{H}, 10.31$. Found $\mathrm{C}, 60.50 ; \mathrm{H}, 10.81$. Active oxygen content: calculated: 0.244 . By iodometric titration: 0.238. After iodometric titration, product was extracted by DCM and NMR analysis revealed that only ketone $\mathbf{1 9}$ was formed.

\section{[bis(tert-butylperoxy)methyl]benzene 43a: ${ }^{11}$}

To a solution of $0.1 \mathrm{mmol}$ of $\mathrm{I}_{2}(25.4 \mathrm{mg})$ and $2 \mathrm{mmol}$ of $60 \%{ }^{t} \mathrm{BuOOH}$ decane solution $(0.72$ $\mathrm{mL})$ in $1 \mathrm{~mL}$ of acetonitrile, $1 \mathrm{mmol}(120 \mathrm{mg})$ of benzaldehyde 39a was added and the solution stirred at $22^{\circ} \mathrm{C}$ for 24 hours. The reaction mixture was then concentrated under reduced pressure (cca. $20 \mathrm{mmHg}$ ), and the product isolated by column chromatography $\left(\mathrm{SiO}_{2}\right.$, petrolether:ether=95:5). Obtained was $101 \mathrm{mg}(38 \%)$ of colorless oil. ${ }^{1} \mathrm{H}$ NMR $\left(\mathrm{CDCl}_{3}\right): 1.27$ $(\mathrm{s}, 18 \mathrm{H}), 6.18(\mathrm{~s}, 1 \mathrm{H}), 7.25-7.50(\mathrm{~m}, 5 \mathrm{H}) ;{ }^{13} \mathrm{C} \mathrm{NMR}\left(\mathrm{CDCl}_{3}, \mathrm{ppm}\right): 26.4,81.1,108.5,127.2$, $128.1,129.0,134.7$.

\section{General procedure for synthesis of hydroperoxyhemiacetals.}

To a solution of $0.1 \mathrm{mmol}$ of $\mathrm{I}_{2}(25.4 \mathrm{mg})$ and $4 \mathrm{mmol}$ of $30 \% \mathrm{H}_{2} \mathrm{O}_{2}(0.45 \mathrm{~mL})$ in $10 \mathrm{~mL}$ of acetonitrile, $1 \mathrm{mmol}(134 \mathrm{mg}$ ) of 3-phenyl-propionaldehyde 44a was added and the solution stirred at $22^{\circ} \mathrm{C}$ for 24 hours. The reaction mixture was concentrated under reduced pressure (cca. $20 \mathrm{mmHg}$ ), dichloromethane $(10 \mathrm{~mL})$ added and the solution dried over $\mathrm{Na}_{2} \mathrm{SO}_{4}$. The solvent was evaporated to leave $153 \mathrm{mg}(91 \%)$ of colorless oil 45a. Its structure was deduced by comparing NMR spectroscopic data with its $t \mathrm{Bu}$-peroxy methoxy analogue ${ }^{12}$ and active oxygen content was determined by iodometric titration after isolation. Further characterization failed as it is not stable, decomposing to the starting aldehyde although refrigerated.

\section{1-Hydroperoxyoctan-1-ol 45b: ${ }^{13}$}

colorless oil, $134 \mathrm{mg}(83 \%),{ }^{1} \mathrm{H}$ NMR $\left(\mathrm{CDCl}_{3}\right): 0.88(\mathrm{t}, J=7 \mathrm{~Hz}, 3 \mathrm{H}), 1.12-1.80(\mathrm{~m}, 14 \mathrm{H})$, $5.16(\mathrm{t}, J=6 \mathrm{~Hz}, 1 \mathrm{H}), 5.18(\mathrm{bs}, 1 \mathrm{H}), 9.34(\mathrm{bs}, 0.5 \mathrm{H}), 10.10(\mathrm{bs}, 0.5 \mathrm{H}) ;{ }^{13} \mathrm{C} \mathrm{NMR}\left(\mathrm{CDCl}_{3}\right)$ : 14.0, 22.6, 24.5, 29.1, 29.3, 31.7, 32.8, 101.8. IR: 3220, 1136, 1089, 1034, 990, 950. 


\section{Synthesis of hydroperoxyacetals.}

To the solution of $0.05 \mathrm{mmol}$ of $\mathrm{I}_{2}(12.7 \mathrm{mg})$ and $1.5 \mathrm{mmol}$ of $30 \% \mathrm{H}_{2} \mathrm{O}_{2}(0.18 \mathrm{~mL})$ in $2 \mathrm{~mL}$ of $\mathrm{MeOH}, 1 \mathrm{mmol}(128 \mathrm{mg})$ of octanal 44b was added and solution stirred at room temperature for 24 hours. Reaction mixture was concentrated under reduced pressure (cca. 20 $\mathrm{mmHg})$, dichloromethane $(10 \mathrm{~mL})$ added and solution dried over $\mathrm{Na}_{2} \mathrm{SO}_{4}$. Solvent was evaporated and obtained $150 \mathrm{mg}$ of reaction mixture. Structure of $\mathbf{4 4 b}$ was determined by comparison of NMR spectroscopic data and ratio between $\mathbf{4 5 b}$ and $\mathbf{4 6 b}$ was also determined by $1 \mathrm{H}$ NMR of reaction mixture.

1-Methoxyoctyl hydroperoxide $46 b^{14}$ (from mixture)

${ }^{1} \mathrm{H} \mathrm{NMR}\left(\mathrm{CDCl}_{3}\right): 4.84(\mathrm{t}, J=5 \mathrm{~Hz}, 1 \mathrm{H}), 3.33(\mathrm{~s}, 3 \mathrm{H}), 0.83-1.77(\mathrm{~m}, 15 \mathrm{H})$

\section{Interconversion of 34 to 2}

To the solution of $0.05 \mathrm{mmol}$ of $\mathrm{I}_{2}(12.7 \mathrm{mg})$ and $2 \mathrm{mmol}$ of $30 \% \mathrm{H}_{2} \mathrm{O}_{2}(0.22 \mathrm{~mL})$ in $5 \mathrm{~mL}$ of acetonitrile, $0.5 \mathrm{mmol}(101 \mathrm{mg})$ of 4-tert-Butyl-1-methoxy-cyclohexyl-hydroperoxide 34 was added and solution stirred at room temperature for 5 hours. Reaction mixture was concentrated under reduced pressure (cca. $20 \mathrm{mmHg}$ ), dichloromethane $(10 \mathrm{~mL})$ added and solution dried over $\mathrm{Na}_{2} \mathrm{SO}_{4}$. Solvent was evaporated and from ${ }^{1} \mathrm{H}$ NMR spectra quantitative conversion to DHP 2 was determined.

When the same reaction was performed without $\mathrm{I}_{2}$ there was no conversion to DHP 2 at all.

\section{Interconversion of 2 to 34}

To the solution of $0.05 \mathrm{mmol}$ of $\mathrm{I}_{2}(12.7 \mathrm{mg})$ in $5 \mathrm{~mL}$ of acetonitrile $0.5 \mathrm{mmol}(102 \mathrm{mg})$ of DHP 34 was added and solution stirred at room temperature for 5 hours. Reaction mixture was concentrated under reduced pressure (cca. $20 \mathrm{mmHg}$ ), dichloromethane $(10 \mathrm{~mL})$ added and solution dried over $\mathrm{Na}_{2} \mathrm{SO}_{4}$. Solvent was evaporated and in ${ }^{1} \mathrm{H}$ NMR spectra was determined that there was no conversion to hydroperoxy ketal 34.

\section{References}

1. Sanderson, J. R.; Zeiler, A. G. Synthesis 1975, 388.

2. Kim, H. S.; Nagai, Y.; Ono, K.; Begum, K.; Wataya, Y.; Hamada, Y.; Tsuchiya, K.; Masuyama, A.; Nojima, M.; McCullough, K. J. J. Med. Chem. 2001, 44, 2357.

3. Solaja, B. A.; Terzic, N.; Pocsfalvi, G.; Gerena, L.; Tinant, B.; Opsenica, D.; Milhous, W. K. J. Med. Chem. 2002, 45, 3331.

4. Zmitek, K.; Zupan, M.; Stavber, S.; Iskra, J. Org. Lett. 2006, 8, 2491.

5. Terent'ev, A. O.; Kutkin, A. V.; Platonov, M. M.; Ogibin, Y. N.; Nikishin, G. I. Tetrahedron Lett. 2003, 44, 7359.

6. Jefford, C. W.; Li, W.; Jaber, A.; Boukouvalas, J. Synth. Commun. 1990, 20, 2589.

7. Todorovic, N. M.; Stefanovic, M.; Tinant, B.; Declercq, J. P.; Makler, M. T.; Solaja, B. A. Steroids 1996, 61, 688.

8. Kim, H. S.; Nagai, Y.; Ono, K.; Begum, K.; Wataya, Y.; Hamada, Y.; Tsuchiya, K.; Masuyama, A.; Nojima, M.; McCullough, K. J. J. Med. Chem. 2001, 44, 2357.

9. Kim, H. S.; Tsuchiya, K.; Shibata, Y.; Wataya, Y.; Ushigoe, Y.; Masuyama, A.; Nojima, M.; McCullough, K. J. J. Chem. Soc., Perkin Trans. 1 1999,1867.

10. Dussault, P. H.; Lee, I. Q.; Lee, H. J.; Lee, R. J.; Niu, Q. J.; Schultz, J. A.; Zope, U. R. J. Org. Chem. 2000, 65, 8407.

11. Fan, Y. L.; Shaw, R. G. J. Chem. Soc., Perkin Trans. 21973 ,1805.

12. Mukaiyama, T.; Kato, J.; Miyoshi, N.; Iwasawa, N. Chem. Lett. 1985,1255.

13. Sulima, L. V.; Franchuk, I. F. Ukr. Khim.Zh. 1972, 38, 333.

14. Griesbaum, K.; Kim, W. S.; Nakamura, N.; Mori, M.; Nojima, M.; Kusabayashi, S. $J$. Org. Chem. 1990, 55, 6153. 


\section{$\because{ }^{20} 0 \overbrace{}^{\text {oot }}$}

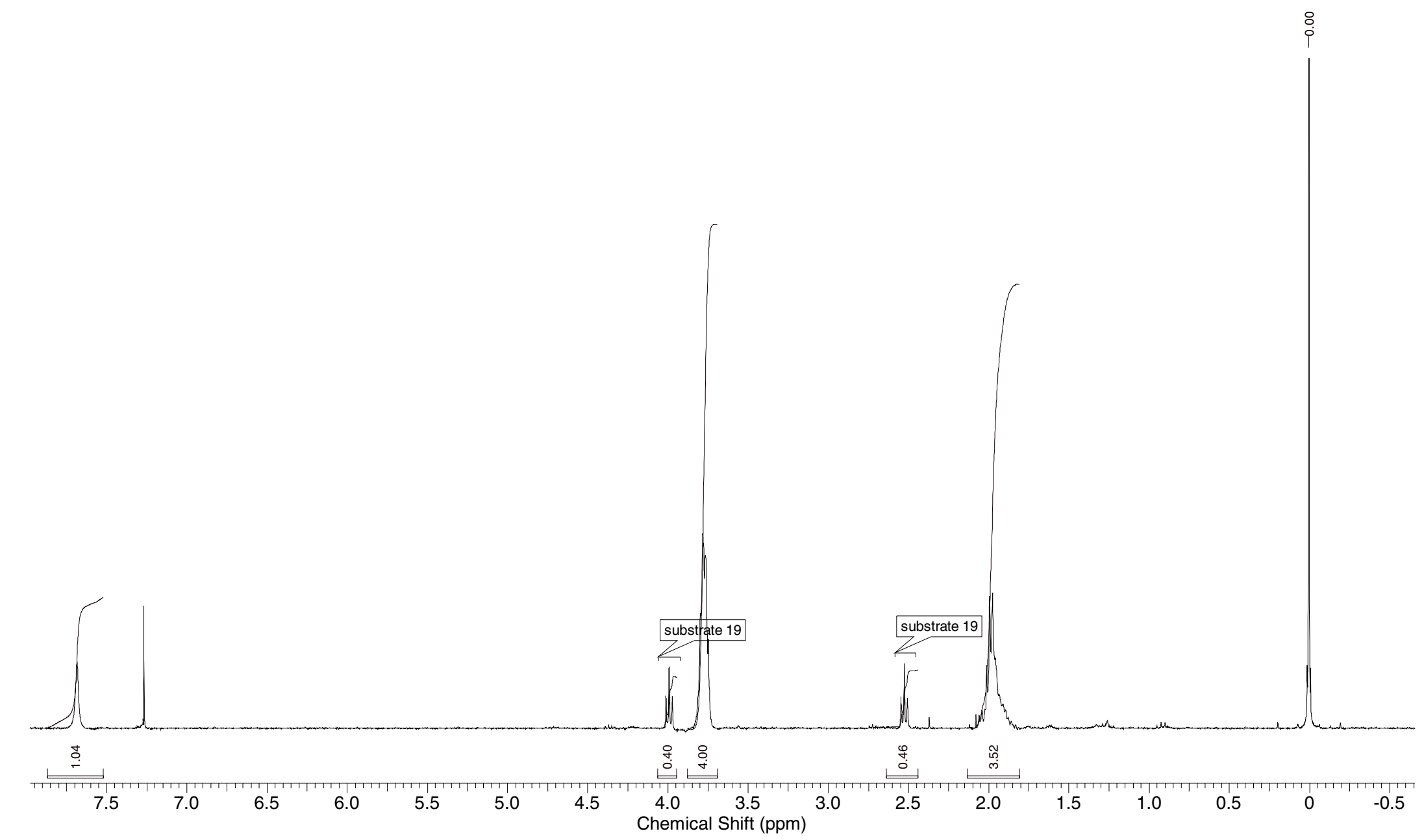




\section{$\because: 0$}

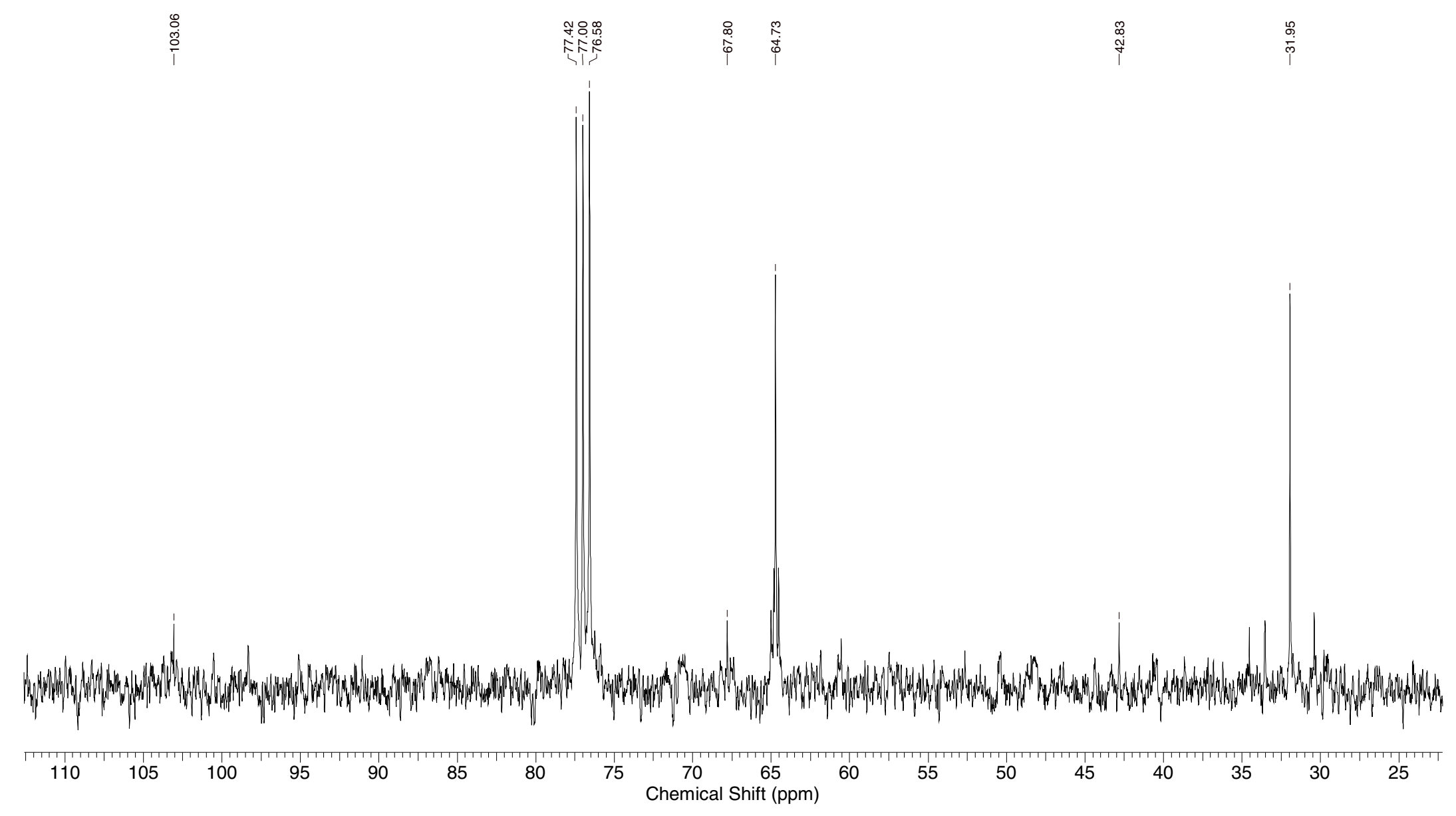




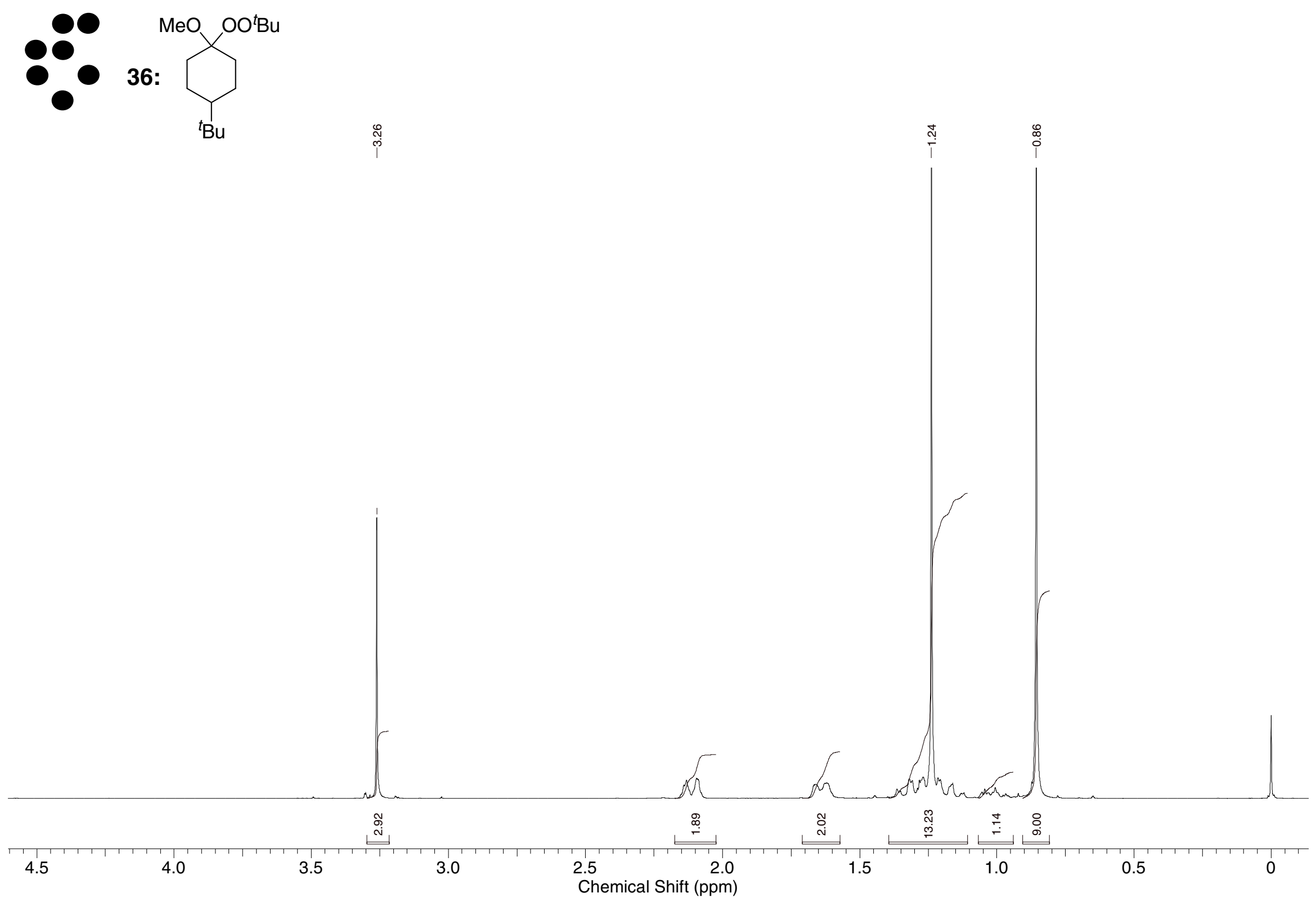




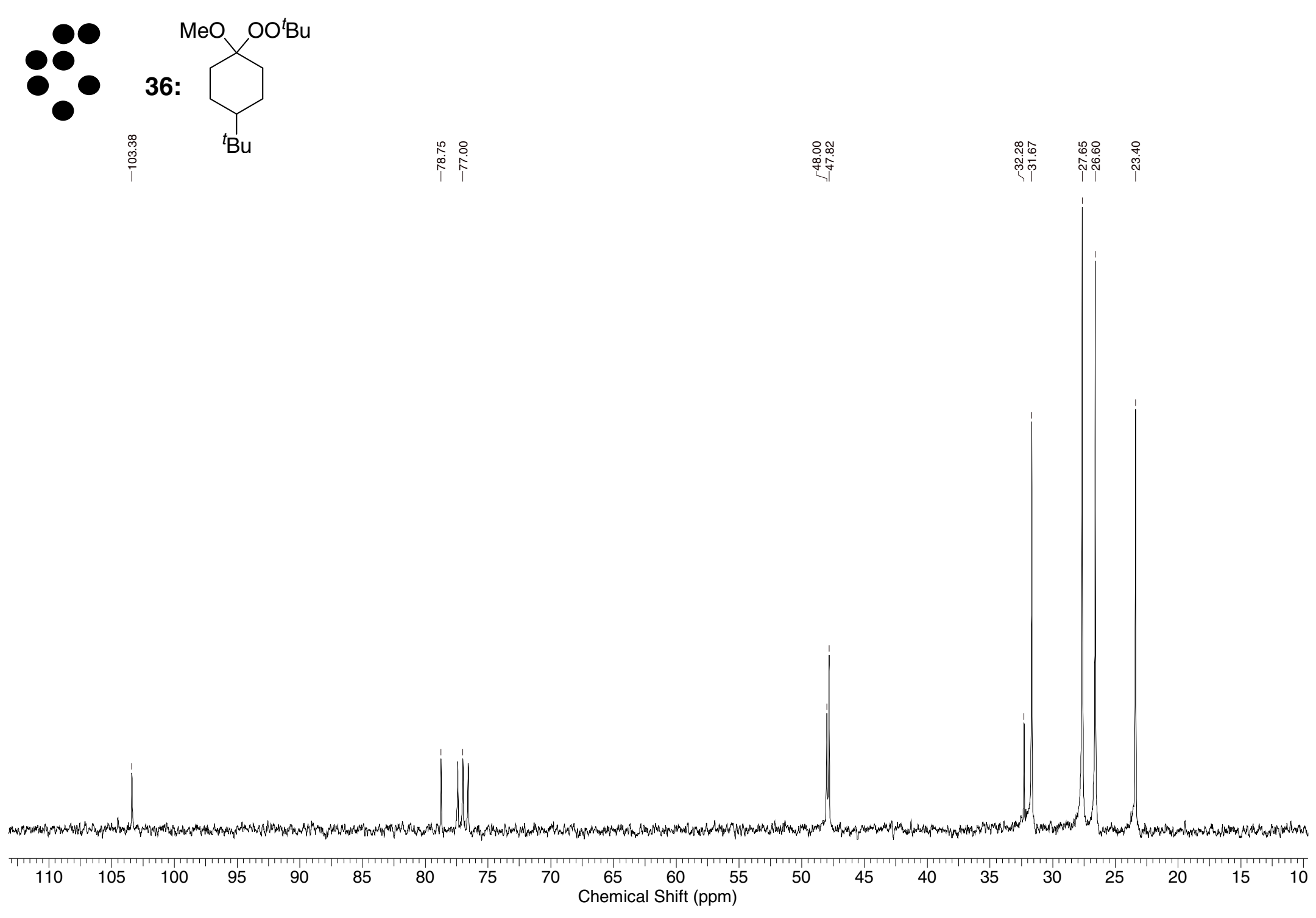




$$
0^{\circ}{ }^{37}{ }_{{ }_{\mathrm{Bu}}}-\chi_{\mathrm{OEt}}^{\mathrm{OOH}}
$$

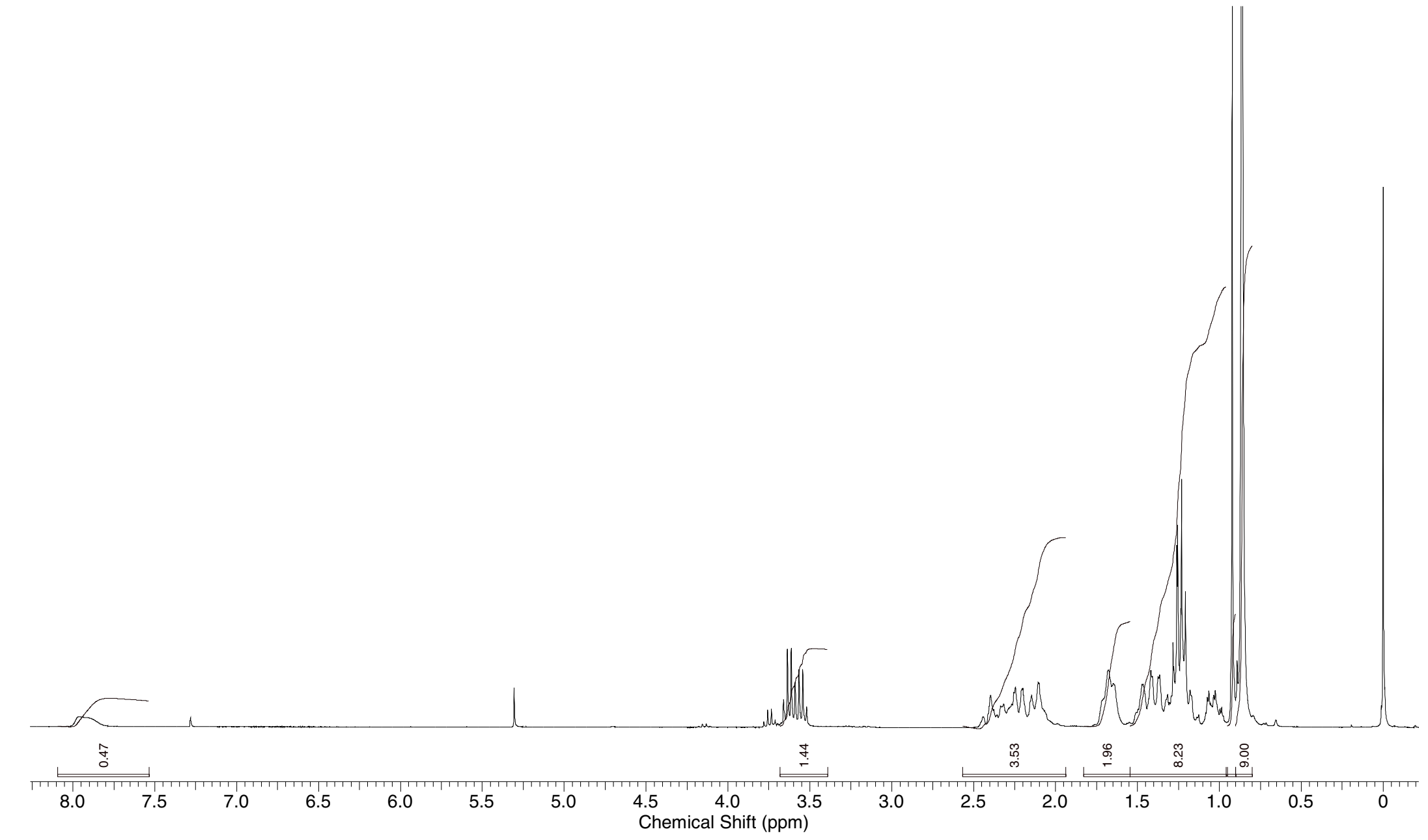




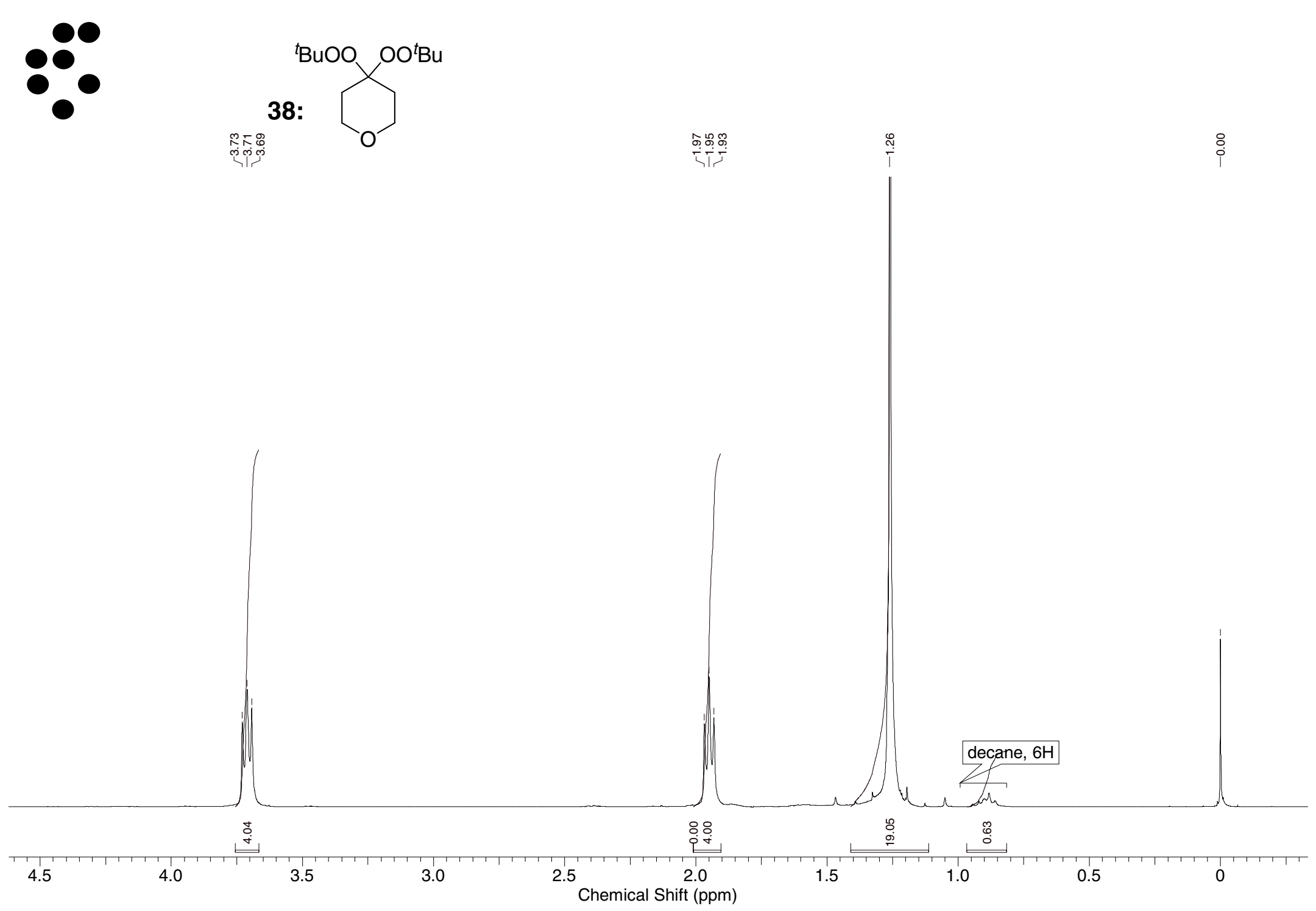




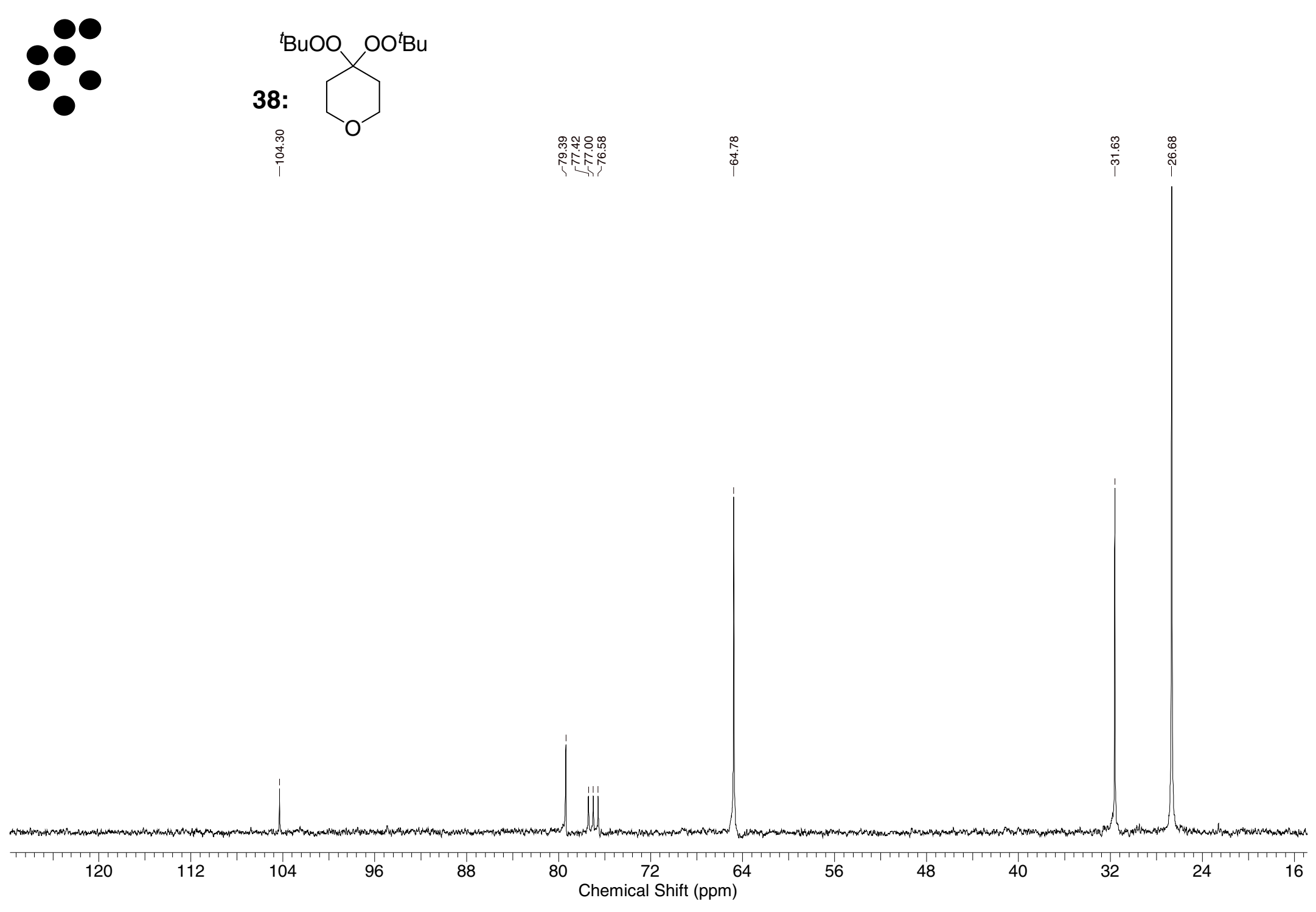


40b:

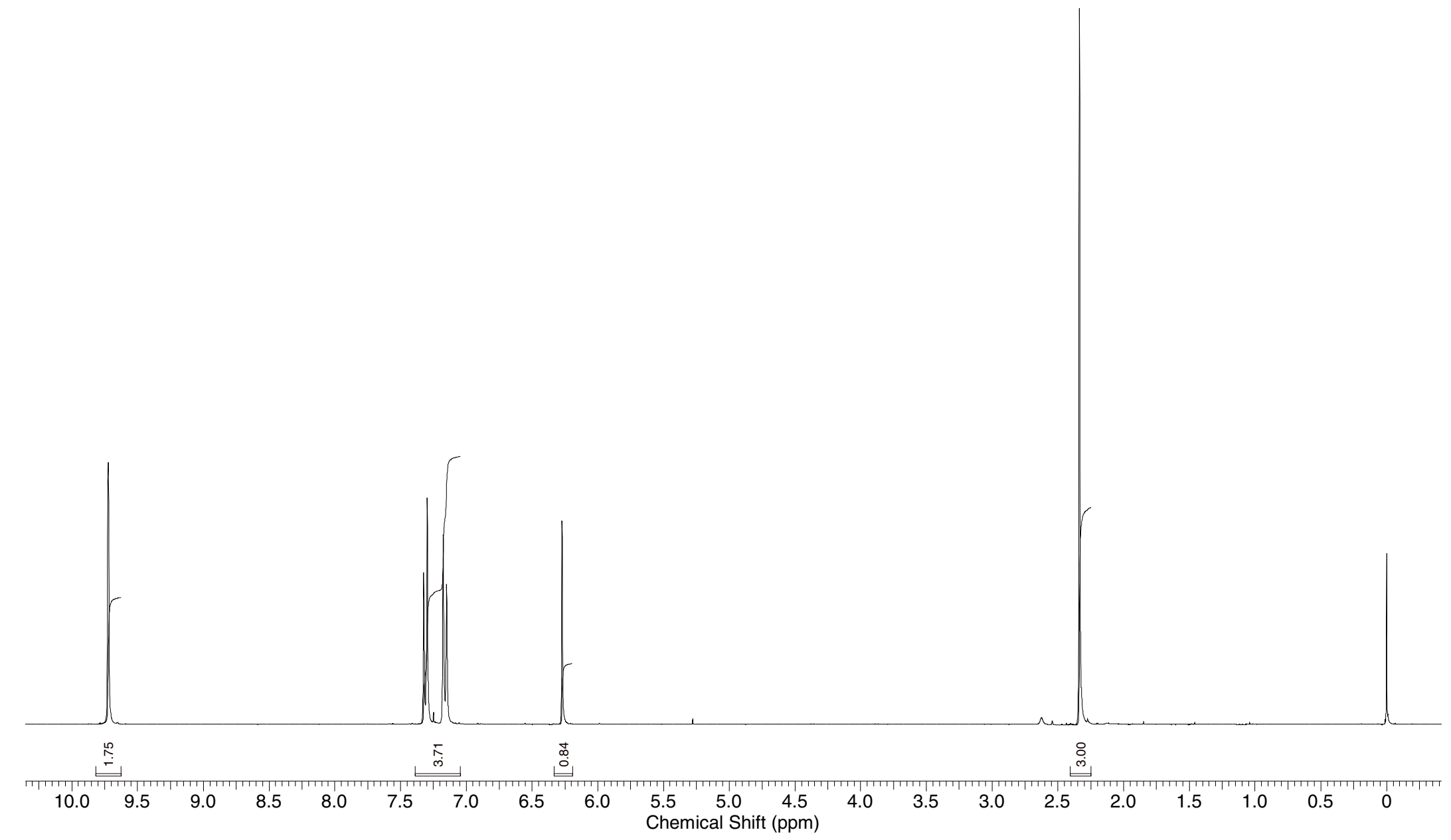



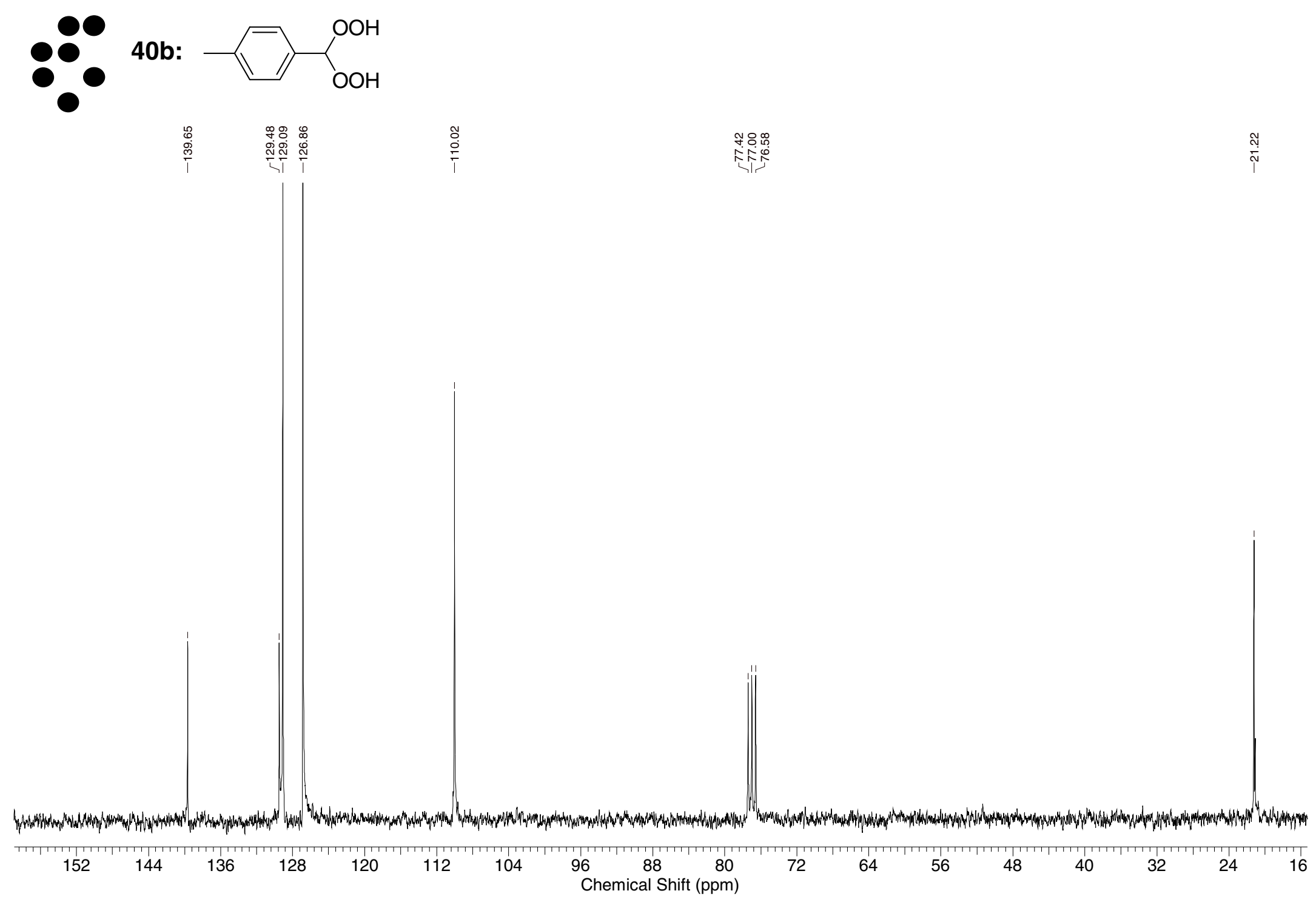
$\because \because 0$

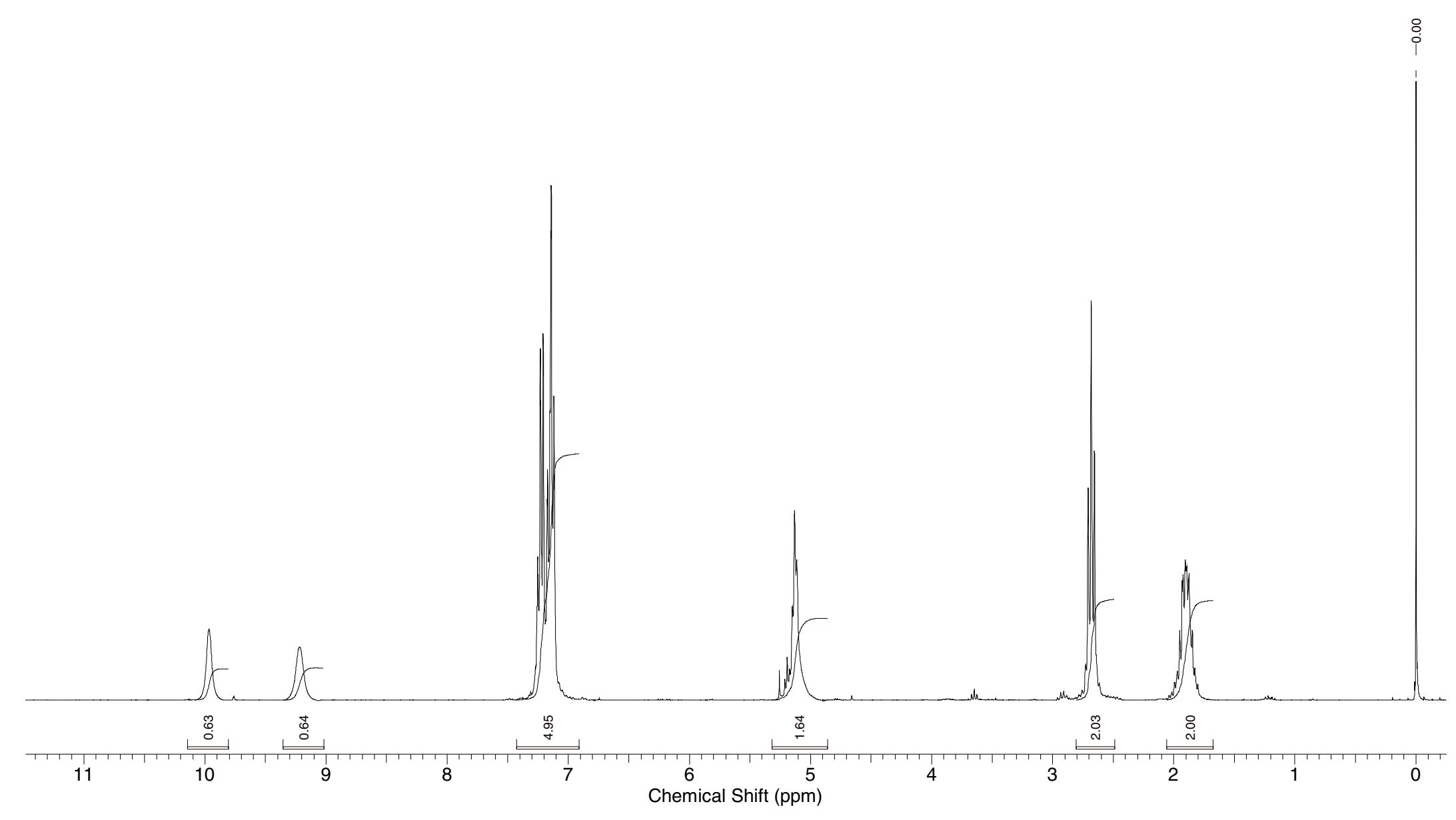




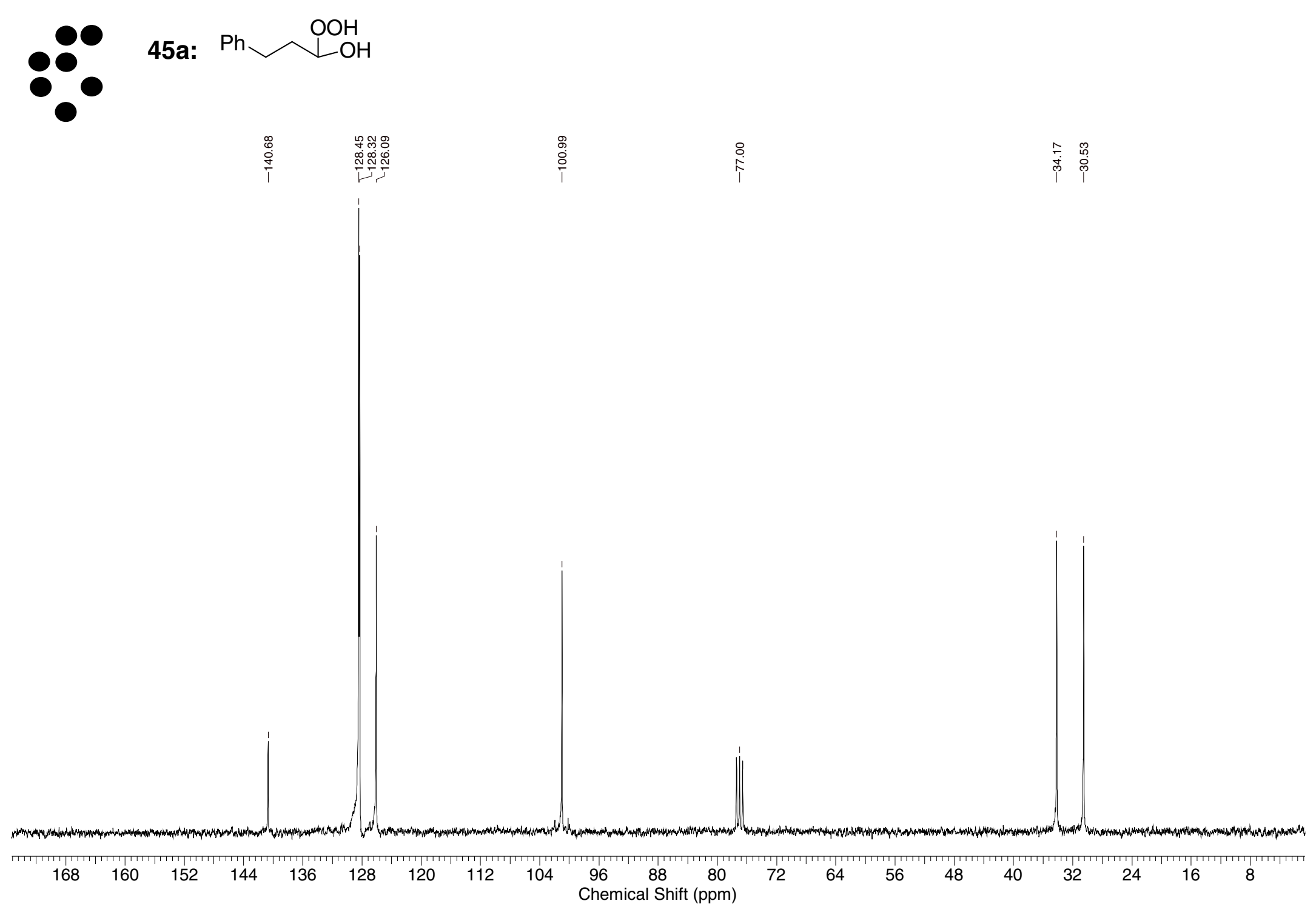


47a: $8^{\circ}$

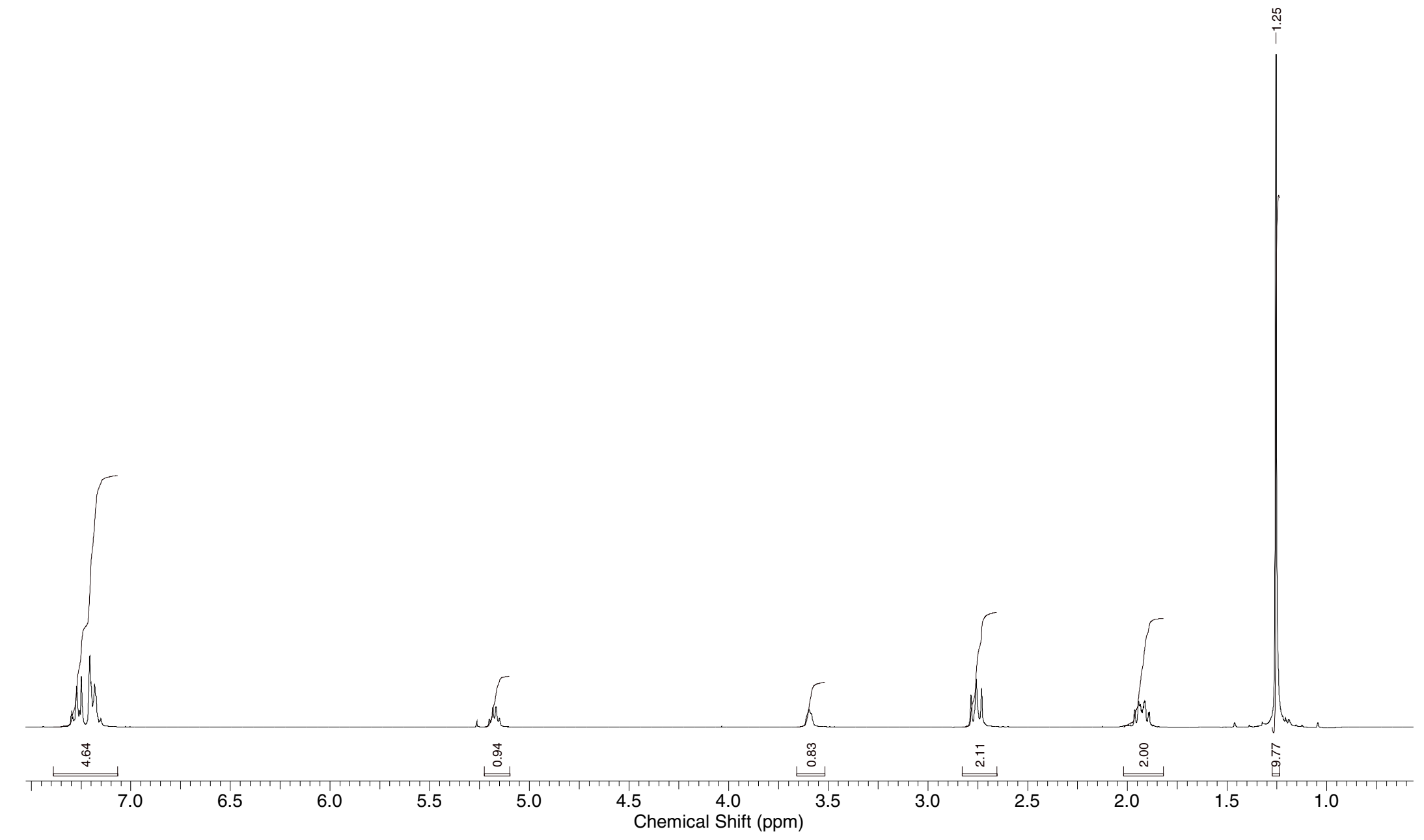




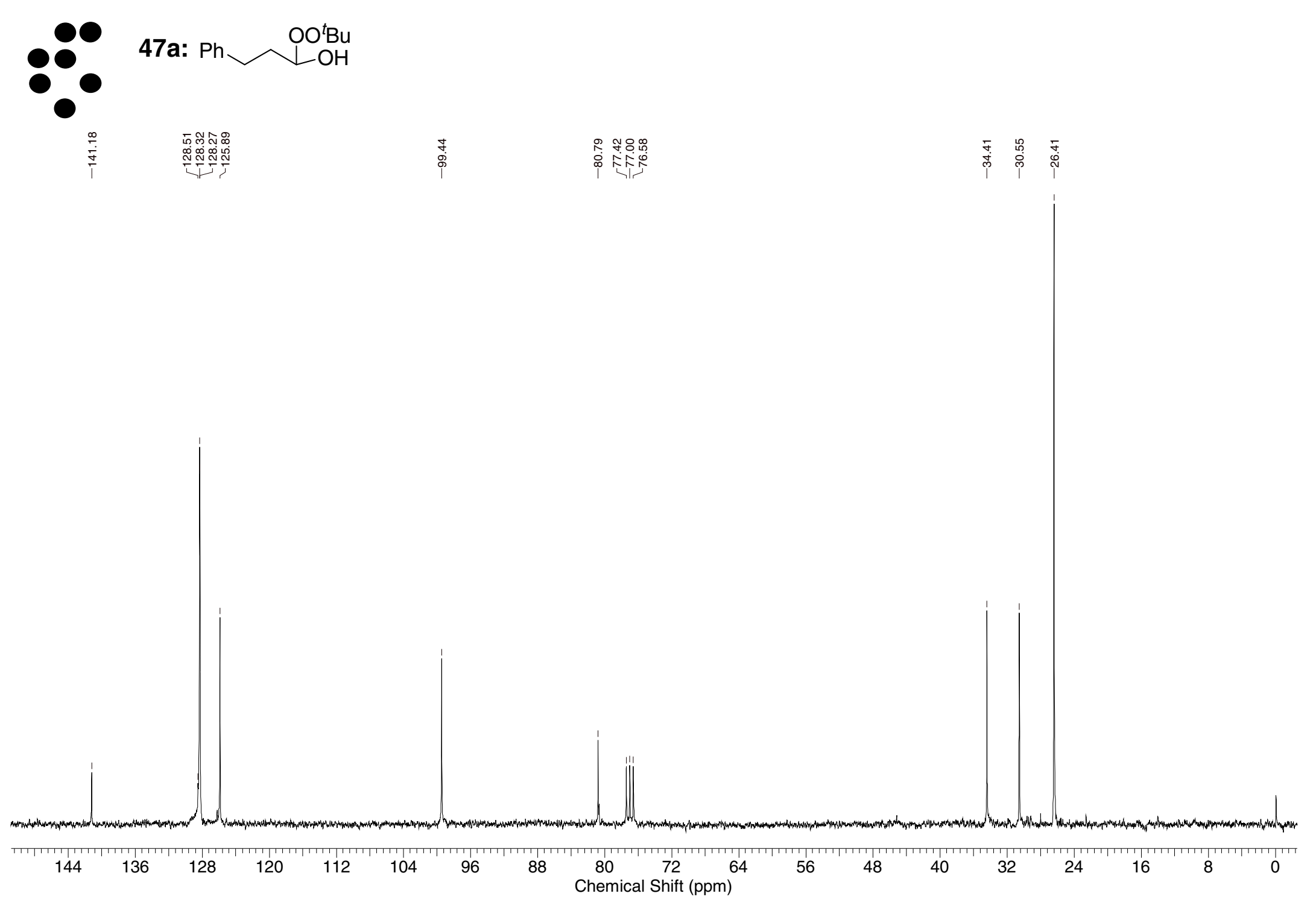

